\title{
Positive Susceptibility Vessel Sign on SWI-MRI Sequence Imaging Might Differentiate Patients Had Silent Brain Ischemia among Apparently Neurologically-Free Patients Yasmin H. Hemeda
}

Department of Radiodiagnosis, Interventional Radiology and Medical Imaging, Faculty of Medicine, Menoufia University, Egypt

Corresponding author: Yasmin H. Hemeda, Mobile: (+20) 01061173115, E-Mail: hosneyyasmin@gmail.com

\begin{abstract}
Background: The susceptibility-weighted imaging (SWI) is an essential MRI sequence in the assessment of acute ischemic stroke. Silent cerebrovascular disease is five times more prevalent than symptomatic brain infarcts and is associated with future risk for stroke and dementia.

Objectives: Evaluation of the diagnostic performance of susceptibility-weighted magnetic resonance sequence imaging (SWI) for early diagnosis of silent brain infarction (SBI) in apparently neurologically-free patients presented by transient neurological manifestations.

Patients and Methods: The study included 218 patients who were clinically evaluated for demographic, clinical data concerning presence of chronic medical diseases, presenting symptoms and its frequency and severity. Routine lab investigations and lipid profile were performed and the plasma atherogenic index (PAI) for oncoming cardiovascular insults was calculated. MRI scan was performed using 1.5 T MRI scanner (Toshiba Vantage) with a head coil.

Results: 102 patients (46.8\%) had chronic medical diseases and hypertension (HTN) and diabetes mellitus (DM) are the most common. The commonest complaint was occasional amnesia, slurred speech and weak handgrip. PAI defined 53 patients at high, 101 patients at intermediate and 64 patients at low risk of cardiovascular insults. Susceptibility vein sign (SVS)+ were detected in 78 SWI scans and showed positive significant correlation with smoking, multiple comorbidities, presence of chronic kidney disease, DM, hypertension and with PAI.

Conclusion: The presence of SVS in SWI during MRI examination is pathognomonic sign for the presence of SBI. The incidence of SBI on SWI scans of apparently neurologically free patients who presented by transient neurological manifestations is high and was found to be associated with the presence of chronic medical diseases especially in obese dyslipidemic patients.
\end{abstract}

Keywords: Apparently neurologically-free patients, Silent brain infarction, Susceptibility-weighted magnetic resonance sequence imaging, Susceptibility vein sign.

\section{INTRODUCTION}

Silent cerebrovascular disease including silent brain infarction (SBI) and white matter disease (WMD) is commonly found incidentally on neuroimaging scans obtained in routine clinical care ${ }^{(1)}$. Silent brain infarction was defined as a brain lesion that is presumably a result of vascular occlusion that was detected incidentally by neuroimaging scans in otherwise healthy subjects or during autopsy (2). According to MRI findings, SBI was defined as asymptomatic, well-defined lesions with a diameter $\geq 3$ $\mathrm{mm}$ with the same signal characteristics as the cerebrospinal fluid on T1- or T2-weighted images ${ }^{(3)}$ and were detected by neuroimaging in up to $20 \%$ of asymptomatic patients ${ }^{(4)}$.

The prevalence of SBI showed racial, ethnic, and age differences; however, in the general population it ranges between 8 and $28 \%$ and is higher in symptomatic stroke patients up to $38 \%{ }^{(5)}$. Multiple risk factors other than age could point to the possibility of the presence or development of SBI in the future and include hypertension, hyperlipidemia, smoking, hyperhomocysteinemia, low high-density lipoprotein, and alcohol consumption ${ }^{(6)}$. Moreover, SBI is the most common neurological complication in sickle cell disease patients ${ }^{(7)}$, patients with a family history or who had current coronary heart diseases ${ }^{(8)}$, and patients with internal carotid atherosclerosis ${ }^{(6)}$ or had prosthetic cardiac valves or stents and maintained on anticoagulants ${ }^{(9)}$.

Epidemiological research showed that silent cerebrovascular disease is five times more prevalent than symptomatic brain infarcts ${ }^{(\mathbf{1 0})}$ and is associated with future risk for stroke and dementia ${ }^{(11)}$, thus representing an important opportunity for stroke prevention $^{(\mathbf{1})}$.

The susceptibility-weighted imaging (SWI) is an essential MRI sequence in the assessment of acute ischemic stroke ${ }^{(\mathbf{1 2})}$. The susceptibility vessel sign (SVS) on gradient echo imaging is defined as a hypointense signal exceeding the diameter of the contralateral artery at the site of the thrombus ${ }^{(13)}$. The appearance of SVS is due to the presence of deoxyhemoglobin secondary to occlusion and decreased oxygenation and leads to local magnetic field inhomogeneity with a subsequent signal loss on the $\mathrm{T} 2 *$ sequences ${ }^{(\mathbf{1 4})}$.

The detection of SVS showed multiple clinical applications, its diameter and length may predict stroke subtype, and longer thrombus size was independently associated with early neurological deterioration in patients with minor stroke and long vessel occlusion following intravenous thrombolysis ${ }^{(15)}$. Moreover, the 
presence of SVS is associated with cardiometabolic disorder subtype ${ }^{(\mathbf{1 6})}$, early recanalization rate in patients with acute ischemic stroke receiving intravenous thrombolysis therapy ${ }^{(14)}$.

The aim of the present work was to evaluation of the diagnostic performance of detection of susceptibility vessel sign on SWI scan for early diagnosis of SBI in apparently neurologically-free patients presented by transient neurological manifestations.

\section{PATIENTS AND METHODS}

This was a prospective cross-sectional study included 218 patients who were clinically evaluated for demographic, clinical data concerning presence of chronic medical diseases, presenting symptoms and its frequency and severity, conducted at diagnostic Radiology Department, Faculty of Medicine, Menoufia University.

The study protocol was designed to include all neurologically-free patients presenting to the outpatients' clinic of the Neurology Department with transient neurological manifestations who were eligible for evaluation. The collected personal and medical history data included age, sex, weight, and height for calculation of body mass index (BMI; $\mathrm{kg} / \mathrm{m}^{2}$ ) and obesity was defined as BMI $\geq 30 \mathrm{~kg} / \mathrm{m}^{2}$, special habits especially smoking, presence of medical morbidities as hypertension (HTN), cardiovascular diseases or previous interventions, diabetes mellitus (DM), chronic kidney disease (CKD), coagulopathy, deep venous thrombosis or varicose veins, history of previous episodes of transient ischemic attacks, maintenance on any medications. Then, patients were clinically examined to evaluate their complaints, regarding their frequency, severity, and associated manifestations. Then, blood samples were obtained for estimation of random blood glucose, liver and kidney functions, and patients were asked to give a blood sample after 12-hr fasting for estimation of lipid profile and calculation of the plasma atherogenic index (PAI) for the possibility of oncoming cardiovascular insults; thereafter patients underwent MRI imaging.

Exclusion criteria: Patients had evident neurological manifestations of acute cerebral infarction, patients with positive findings on MRI other than SVS, contraindication for MRI scan as the presence of a cardiac pacemaker, cardiac prosthetic valves, intracranial aneurysmal clip, or refusal to participate in the study.

Inclusion criteria: Neurologically-free patients who presented with transient neurological manifestations and were free of definite neurological deficits and other exclusion criteria were included in the study.

Methods:
Non-contrast MRI brain was performed using a 1.5 T MRI scanner (Toshiba Vantage, USA) with a head coil. The sequences performed were sagittal T1, axial $\mathrm{T} 2$, diffusion-weighted imaging with $\mathrm{B}$ values of 0 and 1000, Flair weighted image, and SWI. The imaging parameters for SWI were repetition time of $50 \mathrm{~ms}$, time to echo of $40 \mathrm{~ms}$, flip angle $15^{\circ}$, slice thickness $2.5 \mathrm{~mm}$, bandwidth $80 \mathrm{kHz}$, and field of view of $230 \mathrm{~mm}^{2}$, and took an additional 4 minutes of scan time. The SWI sequence generated four sets of images: Phase, magnitude, SWI axial, and thick minimum intensity projection (MIP) images.

\section{MRI evaluation for data collection:}

The following findings in the MRI scan were collected: the location of infarct, presence of dark vessel, prominent cortical and/or intramedullary veins in the vicinity of the infarct. Stroke-associated hemorrhage, if present, was categorized as acute petechial hemorrhage if it occupies $<50 \%$ of the infarct area or macro-hemorrhage if it occupied $>50 \%$ of the infarcted area as compared to the area of diffusion restriction.

\section{Ethical consent:}

An approval of the study was obtained from Menoufia University Academic and Ethical Committee. Every patient signed an informed written consent for acceptance of participation in the study. This work has been carried out in accordance with The Code of Ethics of the World Medical Association (Declaration of Helsinki) for studies involving humans.

\section{Statistical analysis}

Statistical analysis was conducted using IBM ${ }^{\circ}$ SPSS $®$ Statistics (Version 22, 2015; Armonk, USA) for Windows. Obtained data were presented as mean, standard deviation, median, interquartile ranges, numbers, and percentages. Spearman's correlation analysis was used to assess the relationship between positive SVS and demographic, clinical, and lab data. Regression analysis (Stepwise method) was used for stratification of data as a predictor for SVS+ and results were assured using the automatic linear modeling analysis. The positive predictive value of variables was evaluated using receiver operating characteristic (ROC) curve as judged by the area under the curve (AUC). Pvalue $<0.05$ was considered statistically significant.

\section{RESULTS}

421 patients who were presented to Neurology Outpatient clinic with transient neurological manifestations were eligible for evaluation; 203 patients were excluded for not fulfilling the inclusion criteria, and 218 were enrolled in the study and completed the study protocol. Patients' enrolment data are shown in table 1 . 


\begin{tabular}{|l|l|c|c|}
\hline \multicolumn{2}{|l}{ Data } & Number & \% \\
\hline \multirow{4}{*}{ Age (years) } & $<40$ & 37 & 17 \\
\cline { 2 - 4 } & $41-50$ & 68 & 31.2 \\
\cline { 2 - 4 } & $51-60$ & 57 & 26.1 \\
\cline { 2 - 4 } & $>60$ & 56 & 25.7 \\
\cline { 2 - 4 } & Mean \pm SD) & \multicolumn{2}{|c|}{$50.3(9.9)$} \\
\hline \multirow{4}{*}{ Gender } & Male & 131 & 60.1 \\
\cline { 2 - 4 } & Female & 87 & 39.9 \\
\hline \multirow{4}{*}{ BMI $\left(\mathrm{kg} / \mathrm{m}^{2}\right)$} & $<30($ Overweight) & 75 & 34.4 \\
\cline { 2 - 4 } & $30-35($ Obese) & 123 & 56.4 \\
\cline { 2 - 4 } & $>35($ Morbid obese) & 20 & 9.2 \\
\cline { 2 - 4 } & Mean $( \pm$ SD) & & $31.1(2.2)$ \\
\hline \multirow{3}{*}{ Associated morbidities } & Ex-smoker & 65 & 29.8 \\
\cline { 2 - 4 } & Current smoker & 98 & 45 \\
\cline { 2 - 4 } & Never & 55 & 25.2 \\
\cline { 2 - 4 } & Ye & 116 & 53.2 \\
\hline \multirow{3}{*}{$\begin{array}{l}\text { Number of associated } \\
\text { morbidities }\end{array}$} & One & 102 & 46.8 \\
\cline { 2 - 4 } & Two & 38 & 37.3 \\
\cline { 2 - 4 } & Three & 41 & 40.2 \\
\hline \multirow{3}{*}{$\begin{array}{l}\text { Type of associated } \\
\text { morbidities }\end{array}$} & Diabetes mellitus & 23 & 22.5 \\
\cline { 2 - 4 } & Hypertension & 63 & 61.8 \\
\cline { 2 - 4 } & Cardiovascular lesion/surgery & 21 & 20.6 \\
\cline { 2 - 4 } & Chronic kidney disease & 33 & 32.4 \\
\hline
\end{tabular}

Data are presented as numbers, percentage, mean and standard deviation (SD), BMI: Body mass index

All patients presented with transient neurologic manifestations but the commonest complaint was occasional amnesia, slurred speech, and weak handgrip. Mean values of laboratory investigations were within the normally accepted range. However, calculation and categorization of the PAI defined 53 patients $(24.3 \%)$ had a high risk of cardiovascular insults, 101 patients $(46.3 \%)$ had intermediate-risk and 64 patients (29.4\%) had low risk; details of clinical presenting data and laboratory findings are shown in table 2.

Table (2): Clinical presenting complaints and laboratory findings in the studied patients

\begin{tabular}{|c|c|c|c|c|}
\hline \multicolumn{3}{|c|}{ Data } & Number & $\%$ \\
\hline \multirow{10}{*}{$\begin{array}{l}\text { Clinical presenting } \\
\text { complaints }\end{array}$} & \multicolumn{2}{|l|}{ Headache } & 18 & 8.3 \\
\hline & \multicolumn{2}{|l|}{ Dizziness } & 23 & 10.6 \\
\hline & \multicolumn{2}{|l|}{ Vertigo } & 7 & 3.2 \\
\hline & \multicolumn{2}{|l|}{ Slurred speech } & 31 & 14.2 \\
\hline & \multicolumn{2}{|l|}{ Blurred vision } & 13 & 6 \\
\hline & \multicolumn{2}{|l|}{ Muscle fatigue } & 26 & 11.9 \\
\hline & \multicolumn{2}{|l|}{ Weak handgrip } & 29 & 13.3 \\
\hline & \multicolumn{2}{|l|}{ Foot numbness } & 17 & 7.8 \\
\hline & \multicolumn{2}{|l|}{ Amnesia } & 39 & 17.9 \\
\hline & \multicolumn{2}{|c|}{ Lethargy } & 15 & 6.9 \\
\hline \multirow{10}{*}{ Laboratory findings } & \multicolumn{2}{|c|}{ Random blood glucose (mg/dl) } & \multicolumn{2}{|c|}{$88.7 \pm 7.7$} \\
\hline & \multicolumn{2}{|c|}{ Serum urea $(\mathrm{mg} / \mathrm{dl})$} & \multicolumn{2}{|c|}{$26.9 \pm 3.8$} \\
\hline & \multicolumn{2}{|c|}{ Serum creatinine $(\mathrm{mg} / \mathrm{dl})$} & \multicolumn{2}{|c|}{$0.9 \pm 0.14$} \\
\hline & \multicolumn{2}{|c|}{ Serum aspartate transaminase $(\mathrm{mg} / \mathrm{dl})$} & \multicolumn{2}{|c|}{$25.8 \pm 5.4$} \\
\hline & \multicolumn{2}{|c|}{ Serum alanine transaminase $(\mathrm{mg} / \mathrm{dl})$} & \multicolumn{2}{|c|}{$29.2 \pm 6.8$} \\
\hline & \multirow{5}{*}{$\begin{array}{l}\text { Lipid profile } \\
\text { (mg/dl) }\end{array}$} & Total cholesterol & \multicolumn{2}{|c|}{$217.1 \pm 29.2$} \\
\hline & & Triglycerides & \multicolumn{2}{|c|}{$146.7 \pm 20$} \\
\hline & & HDL-c & \multicolumn{2}{|c|}{$42 \pm 5.1$} \\
\hline & & LDL-c & \multicolumn{2}{|c|}{$116.4 \pm 26$} \\
\hline & & VLDL-c & \multicolumn{2}{|c|}{$29.3 \pm 4$} \\
\hline \multirow{4}{*}{\multicolumn{2}{|c|}{ Plasma atherogenic index (PAI) }} & Low risk & 64 & 29.4 \\
\hline & & Intermediate risk & 101 & 46.3 \\
\hline & & High risk & 53 & 24.3 \\
\hline & & Median [IQR] & \multicolumn{2}{|c|}{$0.161[0.11-0.24]$} \\
\hline
\end{tabular}

Data are presented as numbers, percentages, mean, standard deviation, median, and interquartile range (IQR); HDL-c: High-density lipoprotein cholesterol; LDL-c: Low-density lipoprotein cholesterol; VLDL-c: Very low-density lipoprotein cholesterol; low risk: $\mathrm{PAI}=-0.3$ to 0.1 ; intermediate risk: $\mathrm{PAI}=>0.1-0.24$; high risk: $\mathrm{PAI}=>0.24$. 
Evaluation of MRI images and SVS was detected in 78 SWI images; these 78 patients were considered as having SBI, while the remaining 140 patients were considered as both neurologically and radiologically free.

Spearman's correlation analysis showed a positive significant correlation between the incidence of SVS+ and smoking, either current or ex, multiple comorbidities, presence of CKD, DM, HTN, and with PAI, in decreasing order of significance. Stratification of patients' data as regards the importance for the prediction of SBI using the automatic linear modeling analysis defined high PAI as the most important predictor for SVS+ by $48 \%$, smoking by $33 \%$, multiplicity of co-morbidities by $8 \%$, male gender by $7 \%$ and high BMI by $4 \%$ as shown in in table 3 and figure 1.

Regression analysis, stepwise method, defined smoking, and high PAI as the significant predictors for SVS+. Differentiation between high PAI and smoking as the best predictor for SVS+ using ROC curve analysis defined high PAI as the variable with high positive predictive value for SVS+ (Table 3 and figure 2).

Table (3): Statistical analyses of patients demographic, clinical, and laboratory data as predictors for SVS+

\begin{tabular}{|c|c|c|c|c|}
\hline \multicolumn{5}{|c|}{ Spearman's correlation analysis } \\
\hline Variables & \multicolumn{3}{|c|}{ Rho } & $\mathrm{p}$ \\
\hline Age & \multicolumn{3}{|c|}{0.124} & 0.067 \\
\hline BMI & \multicolumn{3}{|c|}{0.111} & 0.107 \\
\hline Male gender & \multicolumn{3}{|c|}{0.127} & 0.061 \\
\hline Smoking & \multicolumn{3}{|c|}{0.431} & $<0.001$ \\
\hline Multiple co-morbidities & \multicolumn{3}{|c|}{0.164} & 0.016 \\
\hline Hypertension & \multicolumn{3}{|c|}{0.141} & 0.039 \\
\hline Cardiac disease/surgery & \multicolumn{3}{|c|}{0.077} & 0.256 \\
\hline Diabetes mellitus & \multicolumn{3}{|c|}{0.143} & 0.035 \\
\hline PAI & \multicolumn{2}{|c|}{0.139} & & 0.040 \\
\hline \multicolumn{5}{|c|}{ The Automatic Linear Modeling Analysis } \\
\hline Variables & \multicolumn{4}{|c|}{ Importance \% } \\
\hline PAI & \multicolumn{4}{|c|}{48} \\
\hline Smoking & \multicolumn{4}{|c|}{33} \\
\hline Multiple co-morbidities & \multicolumn{4}{|c|}{8} \\
\hline Male gender & \multicolumn{4}{|c|}{7} \\
\hline BMI & \multicolumn{4}{|c|}{4} \\
\hline \multicolumn{5}{|c|}{ Regression analysis "Stepwise Method" } \\
\hline Variable & \multicolumn{2}{|r|}{$\beta$} & & $\mathrm{P}$ \\
\hline Smoking & \multicolumn{2}{|c|}{0.374} & & $<0.001$ \\
\hline PAI & \multicolumn{2}{|c|}{0.170} & & 0.007 \\
\hline \multicolumn{5}{|c|}{ Receiver Operating Characteristic Curve Analysis } \\
\hline Variables & AUC & SE & $\mathrm{P}$ & $95 \% \mathrm{CI}$ \\
\hline Smoking & 0.578 & 0.038 & 0.056 & $0.502-0.653$ \\
\hline PAI & 0.759 & 0.033 & $<0.001$ & $0.695-0.823$ \\
\hline
\end{tabular}

Rho: Spearman's coefficient; PAI: Plasma atherogenic index; BMI: Body mass index; AUC: Area under the curve; SE. Standard error; CI: Confidence interval. 
Target: SVS

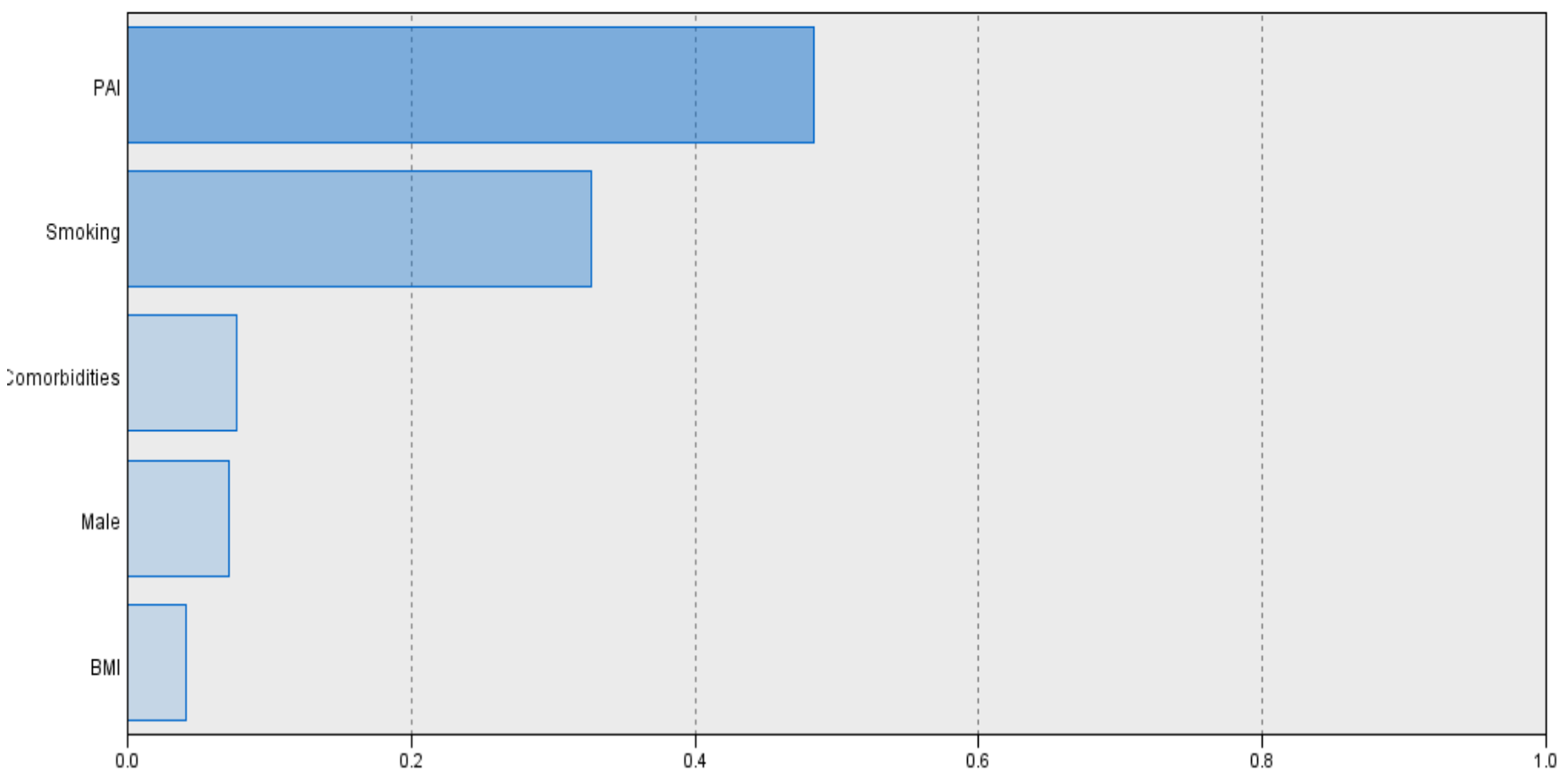

Figure (1): The Automatic Linear Modeling Analysis for the importance of patients' data as predictors for the presence of SVS+ on SWI imaging

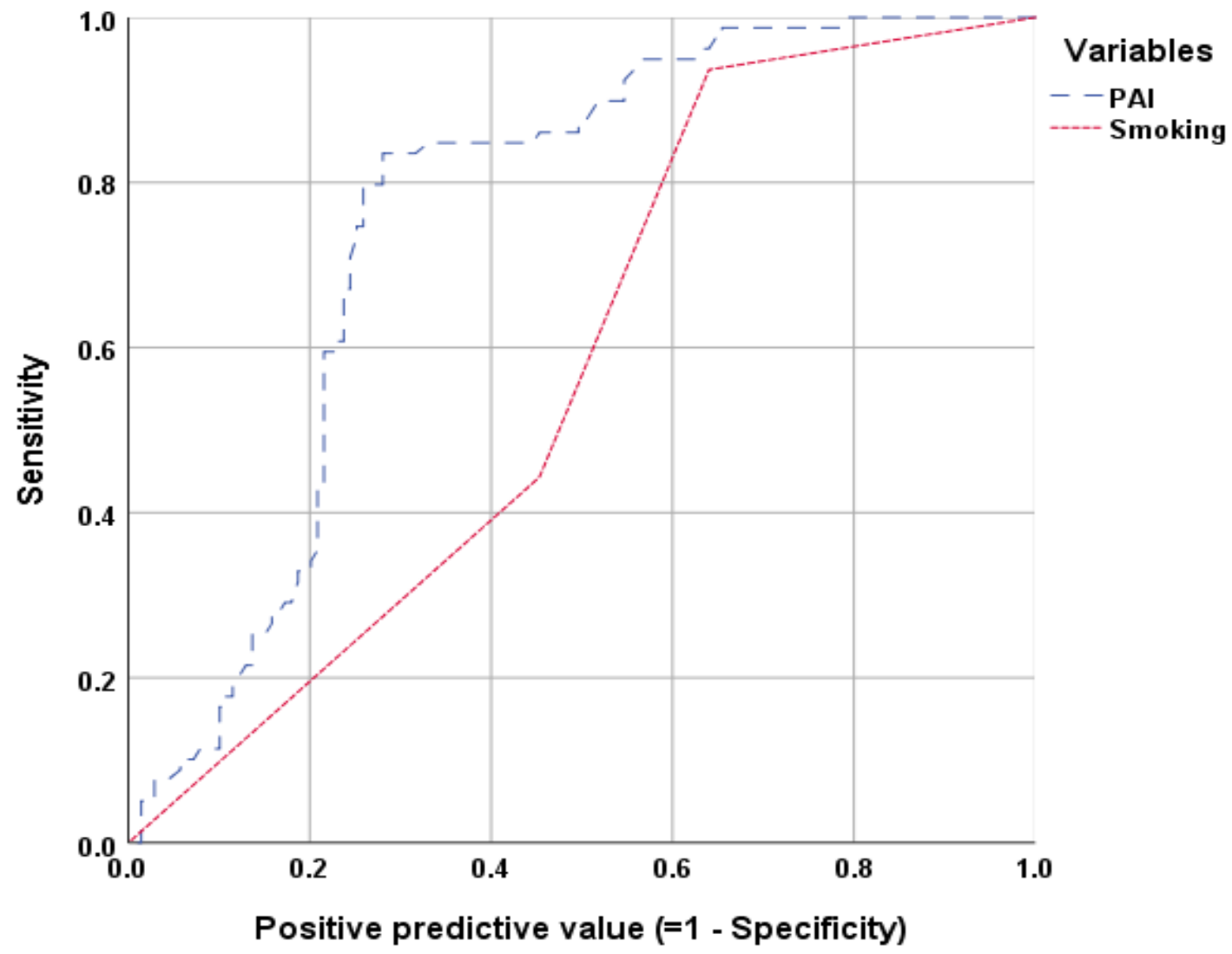

Figure (2): The ROC curve analysis for high PAI and smoking as predictors for the presence of SVS+ on SWI imaging 


\section{Case Presentation}

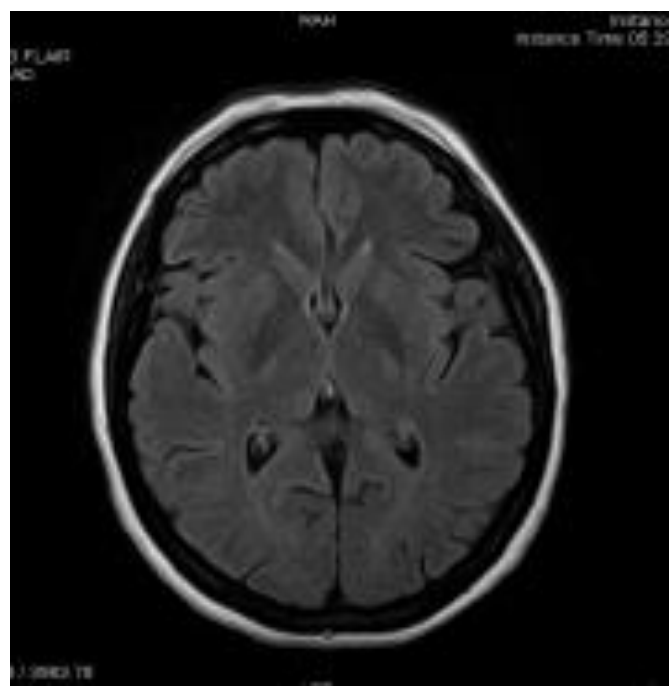

A

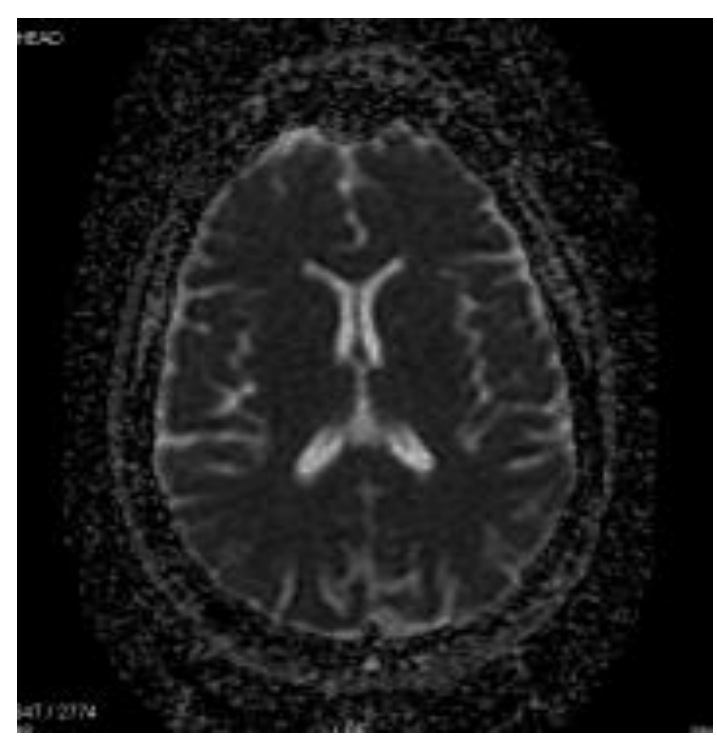

C

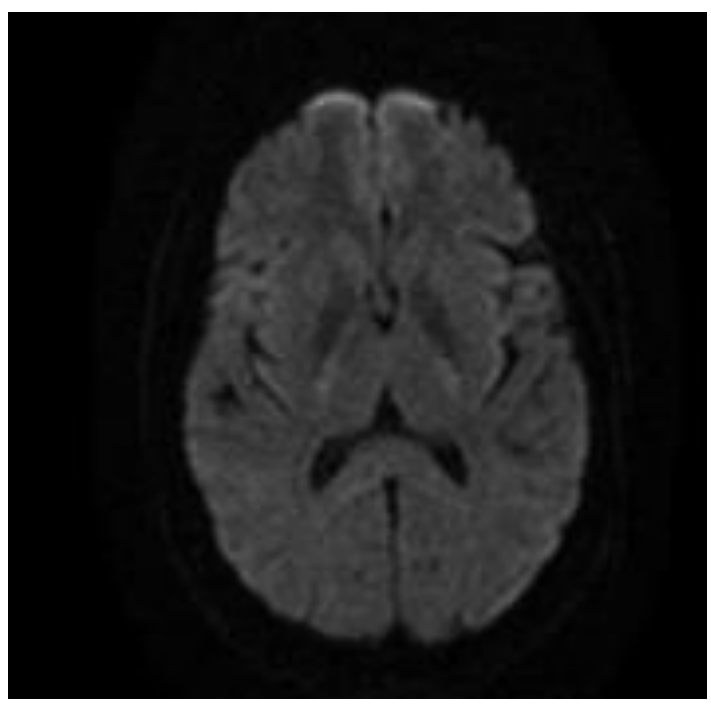

B

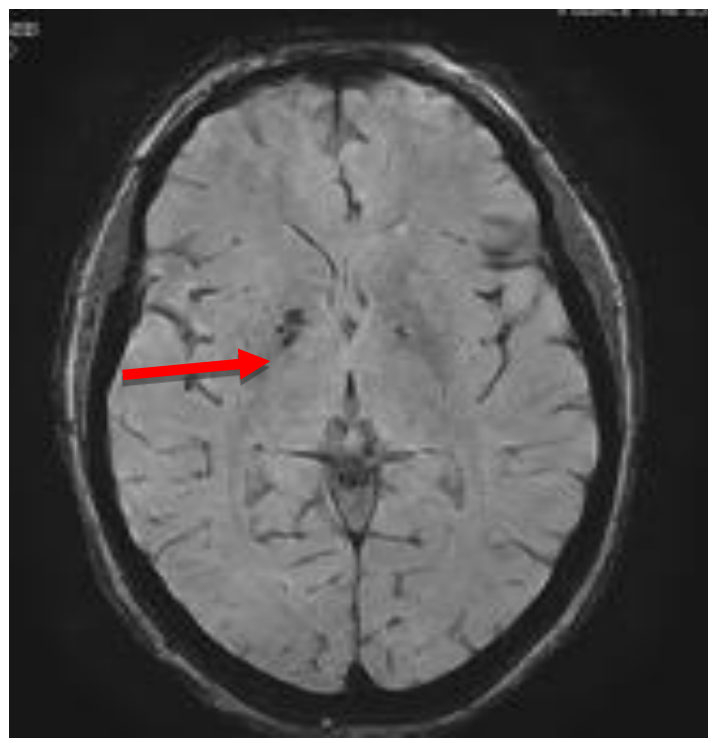

D

Figure (3): A 42-year-old female patient presented with vertigo, dizziness, and numbness of the left lower limb. The fluid-attenuated inversion recovery image of the patient showed no demonstrable hyperintensity (Image A). Diffusionweighted image and ADC were negative with no evidence of restricted diffusion (Image B and C). Susceptibilityweighted image (SWI) showing multiple hypointense vessels at the right lentiform nucleus (Red arrow), denoting increased deoxyhemoglobin concentration (SVS+) that indicated increased oxygen demand of this area and suggestive of SBI. 


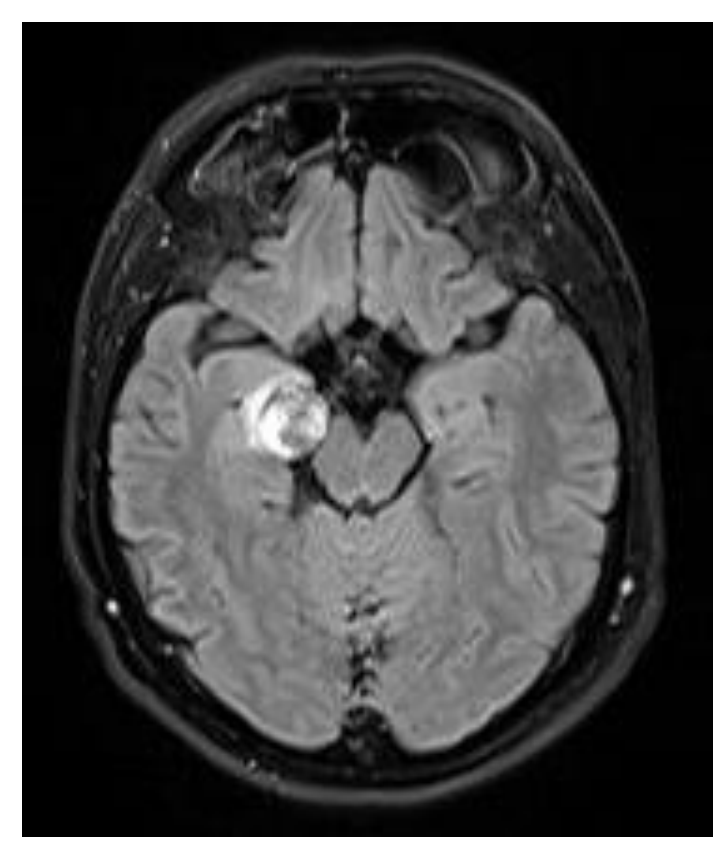

FLAIR Image (A)

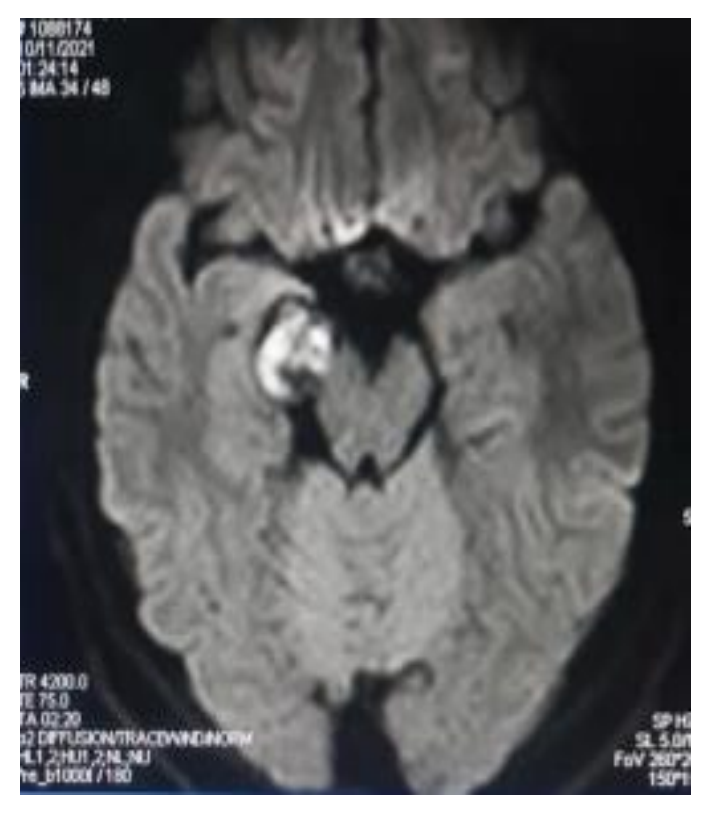

DWI Image (B)

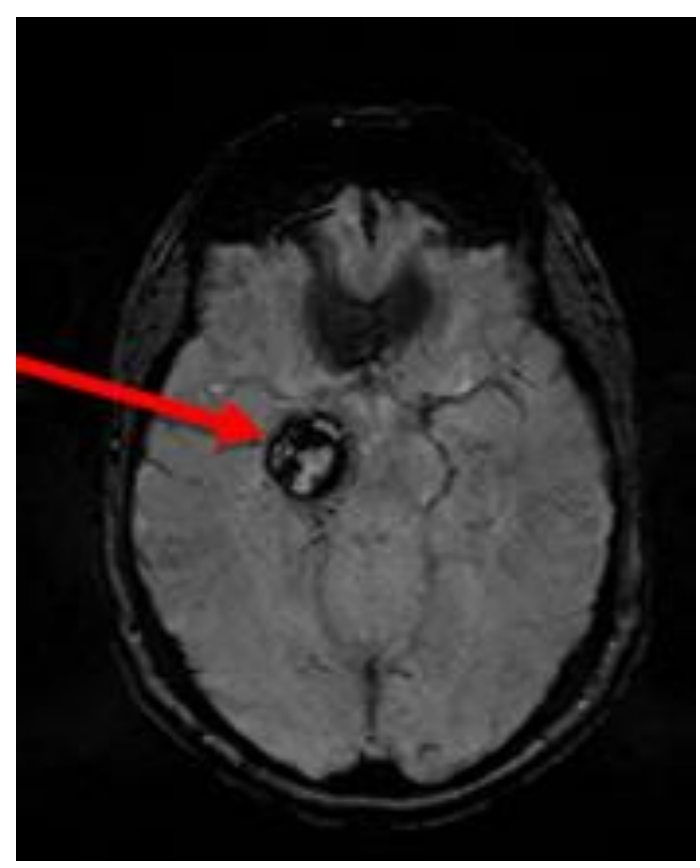

SWI Image (C)

Figure (4): A 41-year-old male patient presented with persistent headaches for 3 hours. The fluid-attenuated inversion recovery image of the patient showed abnormal signal intensity lesion seen at the medial temporal lobe (Image A). Diffusion restriction at (DWI) at the right temporal lobe (Image B). SWI showed vascular anomaly and aneurismal dilatation of the right posterior communicating artery with SVS+ (red arrow) denoting vascular occlusion and intraluminal thrombosis. 

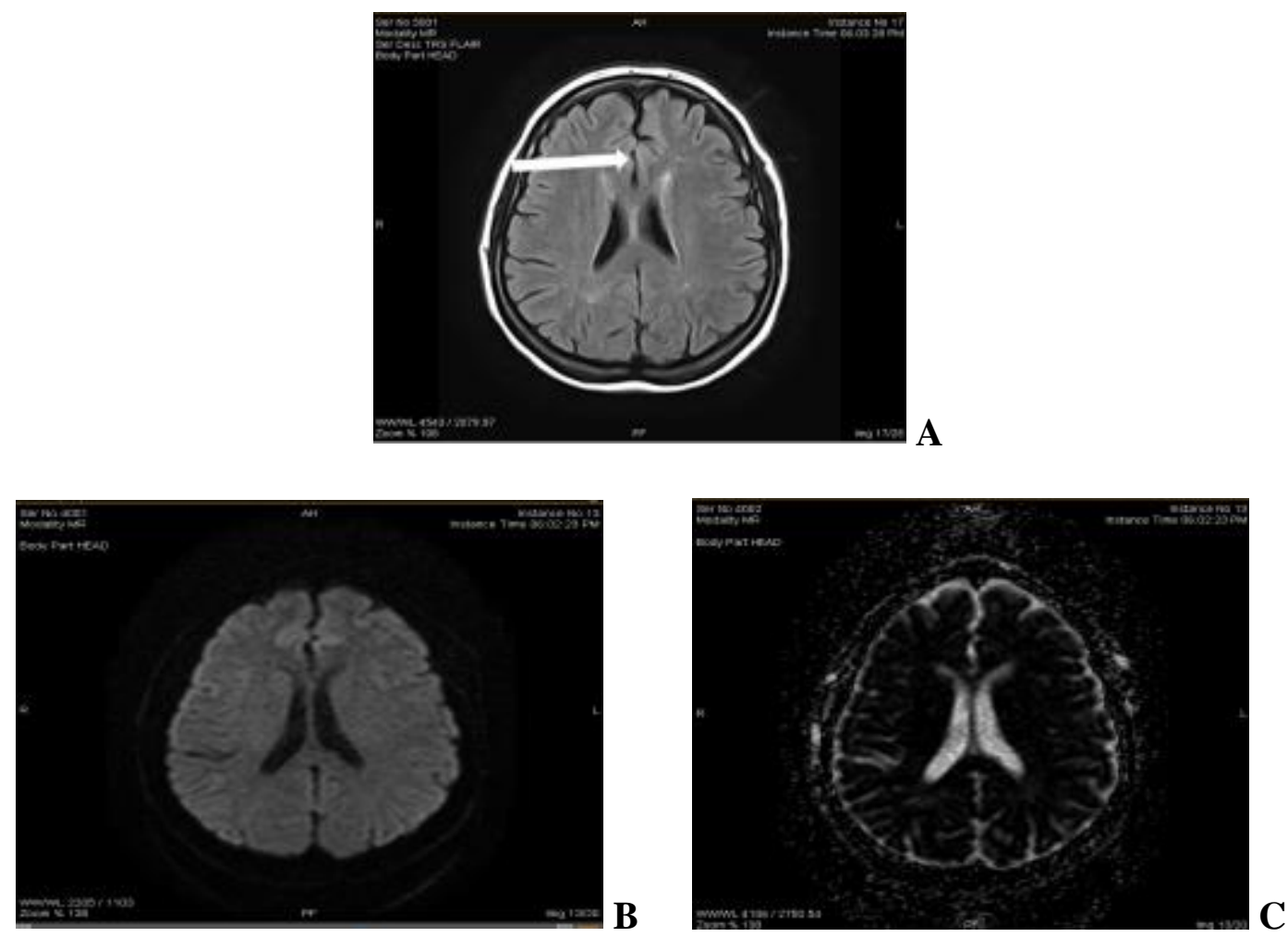

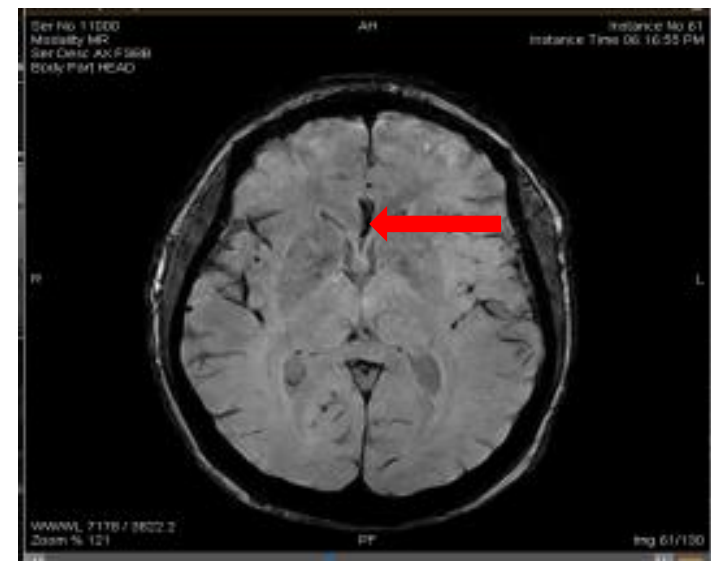

D

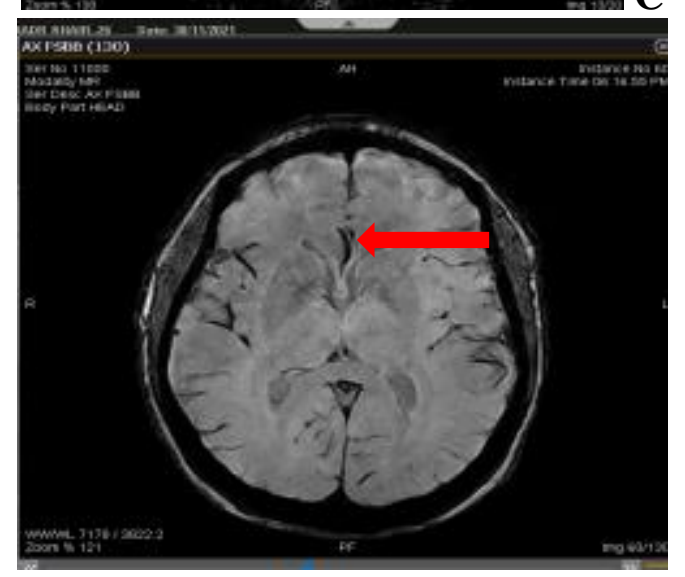

$\mathbf{E}$

Figure (5): A 39-year-old female patient presented with vertigo, dizziness; Fluid-attenuated inversion recovery image of the patient showed slight faint hyperintensity at the frontal cingulate gyrus (White arrow) (Image A). Diffusion weighted image showing slight hyperintensity with negative ADC and no evidence of restricted diffusion (Image B and C). Susceptibility-weighted image (SWI) showing prominent hypointense vessel sign at A2 segment of right anterior cerebral artery (Red arrow), denoting increased deoxyhemoglobin concentration (SVS+) that indicated increased oxygen demand of this area and suggestive of SBI (Image D and E).

\section{DISCUSSION}

The current study assured the feasibility of detection of SVS in SWI images as a pathognomonic sign for incidental detection of SBI in apparently neurologically-free patients presenting by transient neurologic manifestation and allowed for detection of SBI in 78 patients of the studied patients. In line with these data, Yang et al. ${ }^{(17)}$ using SWI and DWI detected higher SBI prevalence in patients with cerebral microbleeds and after 1-year follow up concluded that the presence of SBI in patients undergoing MRI for recent intracranial hemorrhage indicates an elevated risk of future cerebrovascular events and vascular death. Recently, He et al. ${ }^{(18)}$ found the length of the SVS, as measured using the $\mathrm{T} 2 *$ gradient echo imaging, in patients presenting with minor stroke was a powerful independent predictor of early neurological deterioration.

In support of the accuracy of SVS for detection of cerebral insults, Alhazmi et al. ${ }^{(19)}$ found the SVS length can differentiate cardioembolic from large-artery atherosclerosis subtype of embolic stroke of undetermined source with sensitivity and specificity rates of $79.7 \%$ and $72.7 \%$, respectively. Also, Guenego et al. ${ }^{(20)}$ reported that clot shape in SVS as determined on T2* imaging in patients presenting with proximal middle cerebral occlusion can predict the success of reperfusion during mechanical thrombectomy. Moreover, Rudilosso et al. ${ }^{(21)}$ found in patients with recent small subcortical infarcts, detection of SVS is 
uncommon but its presence is associated with a higher grade of deep white matter hyperintensities and brain atrophy, and more lacunae.

Correlation analysis detected a positive nonsignificant correlation between the incidence of SBI and patients' age, a finding indicating the importance of other factors than age for the development of SBI. In line with this finding, Kent $\boldsymbol{e t}$ al. ${ }^{(1)}$ found the hazard risk for incidental detection of SBI during MRI examination was higher for patients $<65$ than patients $>65$ years old.

Analysis of patients' clinical enrolment data detected 102 patients had chronic medical diseases and about $63 \%$ of patients had more than one chronic medical disease with hypertension and diabetes mellitus were the most common among these 102 patients. Moreover, the detection rate of SVS signs showed a positive significant correlation with the multiplicity of associated medical problems and with the presence of hypertension, DM, and chronic kidney diseases. Similarly, Miglinas et al. (22) documented that subclinical cerebrovascular disease as SBI is more prevalent with declining renal function and Pini $\boldsymbol{e t} \boldsymbol{a l}$. (23) reported that male sex, hypertension, dyslipidemia, smoking, contralateral carotid occlusion, and severity of carotid stenosis were associated with SBI in patients with asymptomatic carotid stenosis. Raghavan et al. ${ }^{\text {(24) }}$ found an association between brain ischemic attacks either silent or symptomatic with hypertension and alcohol history

The calculated BMI showed that all of the enrolled patients were either overweight or obese, and 20 patients $(9.2 \%)$ were morbidly obese with BMI $>35$ $\mathrm{kg} / \mathrm{m}^{2}$. Interestingly, the estimated lipid profile and calculated PAI detected 53, 101, and 64 patients who had a high, intermediate, or low risk of cardiovascular events, respectively, irrespective of age with a positive significant correlation between PAI and BMI. Moreover, there was a positive significant correlation between the incidence of SVS+ and the calculated level of PAI, and statistical analyses defined high PAI as the most important predictor for the presence of SVS+. These findings indicated that obesity with associated dyslipidemia with a high TG/HDL ratio is a strong predisposing factor for the development of SBI attacks.

Similarly, Nam et al. ${ }^{(25)}$ in a series of studies of the prevalence of SBI in a neurologically healthy population found high TG/HDL cholesterol ratio, the presence of a metabolic abnormality, and not obesity per se (26), and high visceral adipose tissue (27) are positively associated with the prevalence of SBI in a neurologically healthy population. Thereafter, Woo et al. (28) depending on the presence of silent lacunar infarct and white matter hyperintensities as indices of small-vessel diseases based on brain MRI in neurologically healthy participants detected a positive association between high TG/HDL ratio and the development of intracranial atherosclerosis
These data indicated the importance of MRI evaluation of apparently neurologically-free patients presenting by transient neurological manifestations with special regard to SWI images for detection of SVS, which is a pathognomonic sign for the presence of temporary vascular occlusion that needs rapid and meticulous intervention to safeguard against the development of permanent occlusion and development of manifest cerebral ischemia. In support of this assumption, Ganeshan et al. ${ }^{(29)}$ documented that in a relevant proportion of patients presented by transient global amnesia, MRI reveals acute ischemic cerebral lesions and recommended cerebral MRI as a mandatory diagnostic line for these patients. Moreover, multiple recent studies advocated MRI evaluation of patients undergoing invasive vascular or cardiac procedures for early detection of SBI after this procedure, where Woldendorp et al. ${ }^{(30)}$ detected an incidence of at least one new SBI after diagnostic conventional cerebral angiogram as detected by post-procedural MRI and meta-regression suggested an association between the mean number of new SBI and incidence of early and late post-procedural cognitive dysfunction. Al-Hussain $\boldsymbol{e t}$ al. (31) found diagnostic conventional cerebral angiogram is associated with an incidence of SBI development as judged by MRI performed before and after cerebral angiography. Moreover, Mao et al. ${ }^{(32)}$ and Wieczorek et al. ${ }^{(33)}$ documented that after ablation of atrial fibrillation a significant number of patients still experience silent cerebral embolism, irrespective of the peri-procedural anticoagulation strategy. Furthermore, Hao et al. ${ }^{(34)}$ found the 5-year risks of recurrent stroke and all-cause mortality of cases with silent versus manifest lacunar infarcts that were detected by brain MRI were comparable; $38 \%$ vs $43 \%$ and $11 \%$ vs $14 \%$.

\section{CONCLUSION}

The incidence of SBI on SWI scan during MRI examination of apparently neurologically-free patients who presented by transient neurological manifestations is high and was found to be associated with the presence of chronic medical diseases, especially in obese dyslipidemic patients. The presence of SVS in SWI during MRI examination is a pathognomonic sign for the presence of SBI and could predict its outcome as documented in the literature.

\section{RECOMMENDATIONS}

Transient neurologic manifestations must be seriously evaluated clinically, laboratory and by MRI with special regard to SWI for detection of SVS.

Limitation: Follow-up data of these patients were missed and this limited the evaluation of the predictability of SVS for the outcome.

Acknowledgment: The author thanks staff members of the Neurology Department for case provision and supplementing the study by clinical and lab data of these patients. 
Financial support and sponsorship: Nil. Conflict of interest: Nil.

\section{REFERENCES}

1. Kent D, Leung L, Zhou Y et al. (2021): Association of silent cerebrovascular disease identified using natural language processing and future ischemic stroke. Neurology, 97(13): 1313-1321.

2. Goldberg I, Auriel E, Russell D et al. (2012): Microembolism, silent brain infarcts and dementia. J Neurol Sci., 322(1-2):250-3.

3. Wardlaw J, Smith E, Biessels G et al. (2013): Neuroimaging standards for research into small vessel disease and its contribution to ageing and neurodegeneration. Lancet Neurol., 12(8):822-838.

4. Riba-Llena I, Jarca C, Mundet X et al. (2013): Investigating silent strokes in hypertensives: a magnetic resonance imaging study (ISSYS): rationale and protocol design. BMC Neurol., 13:130-136.

5. Azeem F, Durrani R, Zerna $C$ et al. (2020): Silent brain infarctions and cognition decline: systematic review and metaanalysis. J Neurol., 267(2):502-512.

6. Xu W, Zhang X, Chen $H$ et al. (2021): Prevalence and outcome of young stroke patients with middle cerebral artery stenosis. BMC Neurol., 21(1): 99-103.

7. Houwing M, Grohssteiner R, Dremmen M et al. (2020): Silent cerebral infarcts in patients with sickle cell disease: a systematic review and meta-analysis. BMC Med., 18(1):39396.

8. Johansen M, Nyquist P, Sullivan $K$ et al. (2021): Cerebral small-vessel disease in individuals with a family history of coronary heart disease: The Atherosclerosis Risk in Communities Study. Neuroepidemiology, 55(4):316-322.

9. Özyüncü N, Güleç S, Göksülüik H et al. (2021): Relationship between silent cerebral infarcts and quality of anticoagulation in patients with prosthetic mitral valves. Anatol J Cardiol., 25(3):191-195.

10. Durrani R, Hill M, Smith E (2020): Preventing covert brain infarct-related cognitive impairment and dementia. Can $\mathbf{J}$ Neurol Sci., 47(4):456-463.

11. Smith E, Saposnik G, Biessels G et al. (2017): Prevention of stroke in patients with silent cerebrovascular disease: A scientific statement for healthcare professionals from the American Heart Association/American Stroke Association. Stroke, 48(2): 44-71.

12. Hsu C, Kwan G, Hapugoda S et al. (2017): Susceptibility weighted imaging in acute cerebral ischemia: Review of emerging technical concepts and clinical applications. Neuroradiol J., 30(2):109-119.

13. Brinjikji W, Duffy S, Burrows A et al. (2017): Correlation of imaging and histopathology of thrombi in acute ischemic stroke with etiology and outcome: a systematic review. J Neurointerv Surg., 9(6):529-534.

14. Liu M, Li L, Li G (2019): The different clinical value of susceptibility vessel sign in acute ischemic stroke patients under different interventional therapy: A systematic review and meta-analysis. J Clin Neurosci., 62:72-79.

15. Seners P, Hassen W, Lapergue B et al. (2021): Prediction of early neurological deterioration in individuals with minor stroke and large vessel occlusion intended for intravenous thrombolysis alone. JAMA Neurol., 78(3):321-328.

16. Horie N, Tateishi Y, Morikawa M et al. (2016): Acute stroke with major intracranial vessel occlusion: Characteristics of cardioembolism and atherosclerosis-related in situ stenosis/occlusion. J Clin Neurosci., 32:24-29.
17. Yang K, Feng Y, Mu J et al. (2017): The presence of previous cerebral microbleeds has a negative effect on hypertensive intracerebral hemorrhage recovery. Front Aging Neurosci., 9:49-53.

18. He L, Wang J, Wang F et al. (2021): The length of susceptibility vessel sign predicts early neurological deterioration in minor acute ischemic stroke with large vessel occlusion. BMC Neurol., 21(1):421.

19. Alhazmi H, Bani-Sadr A, Bochaton T et al. (2021): Large vessel cardioembolic stroke and embolic stroke of undetermined source share a common profile of matrix metalloproteinase-9 level and susceptibility vessel sign length. Eur J Neurol., 28(6):1977-1983.

20. Guenego A, Fahed R, Sussman E et al. (2021): Impact of clot shape on successful M1 endovascular reperfusion. Front Neurol., 12: 642877.

21. Rudilosso S, Olivera M, Esteller D et al. (2021): Susceptibility vessel sign in deep perforating arteries in patients with recent small subcortical infarcts. J Stroke Cerebrovasc Dis., 30(1):105-109.

22. Miglinas M, Cesniene U, Janusaite M et al. (2020): Cerebrovascular disease and cognition in chronic kidney disease patients. Front Cardiovasc Med., 7:96-101.

23. Pini R, Faggioli G, Indelicato G et al. (2020): Predictors and consequences of silent brain infarction in patients with asymptomatic carotid stenosis. J Stroke Cerebrovasc Dis., 29(10):105-108.

24. Raghavan S, Graff-Radford J, Scharf E et al. (2021): Study of symptomatic vs. silent brain infarctions on MRI in elderly subjects. Front Neurol., 12:615-19.

25. Nam K, Kwon H, Jeong $\mathrm{H}$ et al. (2019): High triglyceride/HDL cholesterol ratio is associated with silent brain infarcts in a healthy population. BMC Neurol., 19(1):147152.

26. Nam K, Kwon H, Jeong $H$ et al. (2020): Obesity without metabolic disorder and silent brain infarcts in a neurologically healthy population. Int J Obes (Lond), 44(2):362-367.

27. Nam K, Kwon H, Jeong $H$ et al. (2020): Visceral adiposity index is associated with silent brain infarct in a healthy population. Sci Rep., 10(1):17271-75.

28. Woo M, Lee K, Chung D et al. (2021): Triglyceride/HDLcholesterol ratio as an index of intracranial atherosclerosis in nonstroke individuals. Front Neurol., 11:504219.

29. Ganeshan R, Betz M, Scheitz J et al. (2021): Frequency of silent brain infarction in transient global amnesia. https://pubmed.ncbi.nlm.nih.gov/34750675/

30. Woldendorp K, Indja B, Bannon P et al. (2021): Silent brain infarcts and early cognitive outcomes after transcatheter aortic valve implantation: a systematic review and meta-analysis. Eur Heart J., 42(10):1004-1015.

31. Al-Hussain F, Aljafen B, Alhazzani A et al. (2021): Incidence of silent ischemic infarct after diagnostic conventional cerebral angiogram. J Coll Physicians Surg Pak., 31(3):314-317.

32. Mao Y, Wang H, Huang P (2021): Meta-analysis of the safety and efficacy of using minimally interrupted novel oral anticoagulants in patients undergoing catheter ablation for atrial fibrillation. J Interv Card Electrophysiol., 60(3):407-417.

33. Wieczorek M, Bogossian H, Bandorski D et al. (2021): Uninterrupted use of direct oral anticoagulants versus vitamin $\mathrm{K}$ antagonists for catheter ablation of atrial fibrillation with PVAC gold: Incidence of silent cerebral microembolic events. J Interv Card Electrophysiol., 62(1):39-47.

34. Hao Z, Chen Y, Wright $\mathbf{N}$ et al. (2021): Natural history of silent lacunar infarction: 10-year follow-up of a communitybased prospective study of 0.5 million Chinese adults. Lancet Reg Health West Pac., 17: 1-9. 